\title{
ON SOME TREMATODES PARASITES OF FISHES FROM PARANÁ RIVER
}

\author{
FERNANDES, B. M. M. and KOHN, A. \\ Laboratório de Helmintos Parasitos de Peixes, Departamento de Helmintologia, Instituto Oswaldo Cruz, \\ Av. Brasil, 4365, CEP 21045-900, Rio de Janeiro, RJ, Brazil \\ Correspondence to: Berenice M. M. Fernandes, Laboratório de Helmintos Parasitos de Peixes, \\ Departamento de Helmintologia, Instituto Oswaldo Cruz, Av. Brasil, 4365, CEP 21045-900, Rio de Janeiro, \\ RJ, Brazil, e-mail: berenice@ioc.fiocruz.br \\ Received July 5, 2000 - Accepted November 28, 2000 - Distributed August 31, 2001
}

(With 4 figures)

\begin{abstract}
Crassicutis cichlasomae Manter, 1936 is redescribed for the first time in South America and from a new host: Geophagus brasiliensis (Cichlidae). Iheringtrema iheringi Travassos, 1948 is redescribed for the first time since its original description from Pseudopimelodus zungaro (Pimelodidae); and new host records are referred to Genarchella genarchella Travassos et al., 1928, and to Parspina argentinensis (Szidat, 1954). Other reported species are: Microrchis oligovitellum Lunaschi, 1987, Neocladocystis intestinalis (Vaz, 1932), Pseudosellacotyla lutzi (Freitas, 1941), Thometrema overstreeti (Brooks et al., 1979) and Zonocotyle bicaecata Travassos, 1948. Original figures and measurements are presented.
\end{abstract}

Key words: trematodes, freshwater fish parasites, Brazil.

\section{RESUMO}

\section{Sobre alguns trematódeos parasitas de peixes do Rio Paraná}

Crassicutis cichlasomae Manter, 1936 é redescrito pela primeira vez na América do Sul e em um novo hospedeiro: Geophagus brasiliensis (Cichlidae). Iheringtrema iheringi Travassos, 1948 é redescrita pela primeira vez desde sua descrição original, de Pseudopimelodus zungaro (Pimelodidae); novos hospedeiros são referidos para Genarchella genarchella Travassos et al., 1928, e para Parspina argentinensis (Szidat, 1954). Outras espécies estudadas são: Microrchis oligovitellum Lunaschi, 1987, Neocladocystis intestinalis (Vaz, 1932), Pseudosellacotyla lutzi (Freitas, 1941), Thometrema overstreeti (Brooks et al., 1979) e Zonocotyle bicaecata Travassos, 1948. Serão apresentadas figuras originais e medidas.

Palavras-chave: trematódeos, parasitas de peixes de água doce, Brasil.

\section{INTRODUCTION}

A survey of the helminth fauna of fishes from the Paraná River was carried out in 1985, in the locality of Guaíra, Paraná State, South of Brazil. The results concerning the nematodes and some trematodes were already published (Moravec et al., 1992a, b, c, 1993a, b; Kohn \& Fernandes, 1994; Kohn et al., 1997; Kohn et al., 1999). The present paper comprises the results concerning the other digeneans collected in this expedition. Redescription are presented to Crassicutis cichlasomae reported in new host and for the first time in South America and to Iheringtrema iheringi, the first report since its original description. To the other well known species, only the main measurements and comments are presented.

\section{MATERIAL AND METHODS}

Fifty one different species of fishes of a total of 145 specimens were examined from Paraná River, in the locality of Guaíra, Paraná State, Brazil. The used methodology were described in Kohn \& Fernandes (1994). Measuremensts are given in micrometres otherwise stated with means in 
parentheses. Voucher specimens are deposited in the Helminthological Collection of the Oswaldo Cruz Institute (CHIOC). The fish species were classified by Dr. M. P. de Godoy and updated based on Eschmeyer et al. (1998).

\section{RESULTS AND REMARKS}

\section{Crassicutis cichlasomae Manter, 1936 (Homalometridae) (Figs. 1 and 2)}

Redescription and measurements based on 7 whole-mounted worms: body oval 2.11-3.89 mm (3.10) long $\times 1.01-2.00 \mathrm{~mm}$ (1.54) wide. Tegument smooth. Oral sucker subterminal, spherical, 190-330 $(268) \times 190-340$ (276). Ventral sucker pre-equatorial, 220-360 (296) in diameter; sucker width ratio 1:11.2. Prepharynx short 20-90 (43) long. Pharynx 100$170(135) \times 90-210$ (149). Oesophagus 30-130 (71) long. Caeca narrow, overlapping testes, not reaching the posterior extremity. Seminal vesicle sac-shaped, elongate, narrow, reaching ovarian level, measuring 140-420 (257) ×70-120 (89); distal part tubular, opening into a genital pore median, anterior to ventral sucker.

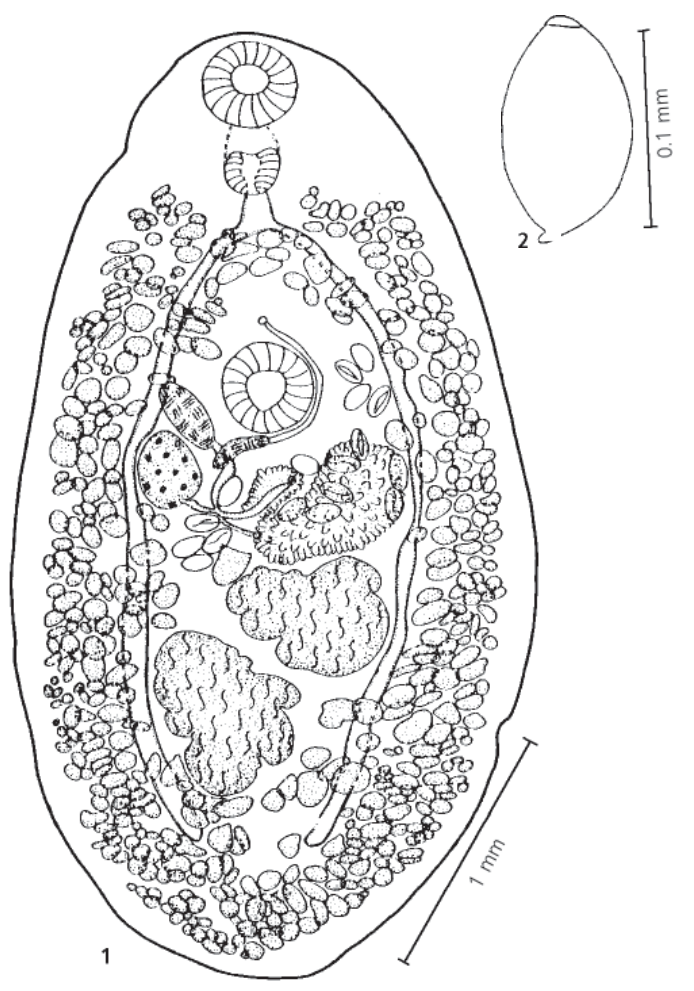

Figs. 1-2 - Crassicutis cichlasomae. 1 - Total, ventral view; 2 - Egg. Original figures.
Testes large, slightly lobated, post-equatorial, intercaecal, close together, diagonal; anterior testis $280-470(380) \times 340-660(490)$; posterior testis 280 $650(510) \times 340-680(488)$. Ovary rounded, pre-testicular, posterolateral to ventral sucker, 130-290 $(222) \times 140-270$ (206). Mehlis' gland well developed $90-510(292) \times 120-620(367)$, opposite to ovary. Seminal receptacle pre-ovarian measuring $190-260 \times 70$ 140. Laurer's canal well evidenciated dorsal, surrounded by glands. Vitelline follicles extracaecal, with few follicles caecal and intercaecal, extending from level of esophageal bifurcation to posterior extremity, confluent in esophageal and posterior regions. Uterus intercaecal, short, with few operculated eggs, $101-139 \times 60-109(124 \times 78)$, provided with small knob. Excretory pore terminal. Excretory vesicle not observed.

Host: Geophagus brasiliensis (Quoy \& Gaimard, 1824), Cichlidae.

Site: intestine.

Material deposited: CHIOC n. 33937 a-g

Intensity of infection: two out of three fish examined were parasitized by seven trematodes each.

In South America the genus Crassicutis Manter, 1936 is represented by C. chuscoi (Pearse, 1920) and C. wallini (Pearse, 1920) in Venezuela, and by $C$. intermedius (Szidat, 1954) in Argentina (Szidat, 1954), Brazil (Kohn \& Fróes, 1986) and Paraguay (Bray et al., 1996). Crassicutis cichlasomae was described by Manter (1936) from the stomach of Cichlasoma mayorum from Mexico; Scholz et al. (1995a) described the life cycle and, referred other hosts in Mexico and Central America. In this opportunity we report $C$. cichlasomae for the first time in South America and in a new host: Geophagus brasiliensis. The specimens studied are larger than those of the original description (2.11-3.89 $\mathrm{mm} \times 1.01-2.00 \mathrm{~mm}$ instead of 0.88$1.29 \mathrm{~mm} \times 0.48-0.83 \mathrm{~mm}$ ) and are more close related to the specimens studied by Scholz et al. (1995) (0.94-2.04 mm $\times 0.54-1.20 \mathrm{~mm})$.

\section{Iheringtrema iheringi Travassos, 1948 (Cryptogonimidae) (Fig. 3)}

Redescription based on 2 specimens: body elongate $2.41-2.82 \mathrm{~mm}$ long by $0.65-0.71 \mathrm{~mm}$ wide. Tegument covered by scales till the level of ovary. Oral sucker spherical, well developed, 260-280 $\times$ 290-300. Ventral sucker pre-equatorial 180-200 in diameter. Sucker wide ratio 1:0.6-0.7. Prepharynx short. Pharynx 120-150 × 120-130. Oesophagus short. Caeca reaching posterior extremity. Cirrus sac 
absent. Seminal vesicle behind ventral sucker, tubular, long, convoluted. Genital pore immediately preacetabular. Testes 9 , smooth, in posterior half of body, measuring 170-220 × 150-220. Ovary 290-350 × 390-470, strongly lobed, median, at level of anterior testis. Vitelline follicles lateral from the level of ventral sucker confluent from post-ovarian region to posterior extremity. Uterus median, intercaecal, from ovary to genital pore. Eggs 38-45 × 19-23.

Host: Pseudopimelodus zungaro (Humbold, 1833) (= Pseudopimelodus roosevelti Borodin, 1927) (Pimelodidae).

Site: intestine.

Material deposited: CHIOC n. 33927 a-b.

Intensity of infection: one out of two fish examined harbored two trematodes.

Travassos (1948b) erected the genus Iheringtrema to the species I. iheringi Travassos, 1948, based on two specimens collected from the intestine of $P$. roosevelti from Mogi-Guaçu River, São Paulo State, Brazil. This is the first report of this trematode since its original description, from the same host from another locality. The specimens studied are smaller (2.41-2.82 $\mathrm{mm} \times 0.65-0.71 \mathrm{~mm}$ instead of $3.5-4 \mathrm{~mm} \times 1-1.2 \mathrm{~mm}$ ) and the vitellaria does not reach the level of the pharynx as in type material.

\section{Microrchis oligovitellum Lunaschi,1987 (Paramphistomidae) (Fig. 4)}

Main measurements based on 2 specimens: body $6.21-7.48 \mathrm{~mm}$ long by $2.52-2.63 \mathrm{~mm}$ wide; oral sucker 680-760 × 650-720; ventral sucker $1.42 \mathrm{~mm} \times$ 1.31-1.35 mm; sucker width ratio 1:1.8-2; oesophagus 340-830 long; pseudo cirrus-sac $790 \times 250$; testes $160-250 \times 220-280$; ovary 380-470 × 350-440; eggs 60-67 × 30-37.

Host: Parauchenipterus galeatus (Linnaeus, 1766) (Auchenipteridae).

Site: intestine.

Material deposited: CHIOC n. 33924 a-b

Intensity of infection: one fish examined was parasitized by two trematodes.

Microrchis oligovitellum was described by Lunaschi (1987) from the intestine of Luciopimelodus pati and Trachycorystes striatulus from Argentina. In Brazil, Pavanelli et al. (1997a) published the histopatology of the infection of $P$. galeatus by $M$. oligovitellum without description of the parasite. Our specimens agree with the original description, although presenting larger body $(6.21-7.48 \mathrm{~mm} \times$ 2.52-2.63 mm instead of 2.33-3.89 $\mathrm{mm} \times 0.86-1.05 \mathrm{~mm}$ ).

\section{Genarchella genarchella Travassos, Artigas \& Pereira, 1928 (Derogenidae)}

Main measurements: body $2.41 \mathrm{~mm}$ long by $0.86 \mathrm{~mm}$ wide; oral sucker $250 \times 270$; pharynx $79 \times$ 94 ; ventral sucker $440 \times 470$; sucker width ratio 1:1.7; seminal vesicle $140 \times 60$; testes $270-300 \times 200$ 290 ; ovary $180 \times 240$; eggs $40-45 \times 19-21$, with one long polar filament.

Host: Pimelodus ornatus Kner, 1858 (Pimelodidae).

Material deposited: CHIOC n. 33923.

Site: stomach.

Intensity of infection: one out of two fish examined were parasitized by one trematode.

The type-material of Genarchella genarchella was reestudied by Kohn \& Fernandes (1988), Kohn et al. (1990) and Scholz et al. (1995b).

Hamann (1989) presented the measurements of G. genarchella from several hosts from Paraná River, Corrientes, Argentina.

In this opportunity a new host, Pimelodus ornatus, is presented for G. genarchella.

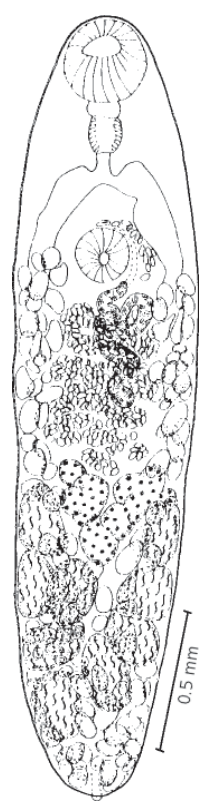

Fig. 3 - Iheringtrema iheringi. Total, dorsal view. Original figure. 
Parspina argentinensis (Szidat, 1954) Sogandares-Bernal, 1959 (Cryptogonimidae)

Main measurements based on 2 wholemounts: body 2.11-2.22 mm long by 0.56-0.71 mm wide; oral sucker $280 \times 250-260$; ventral sucker 240 $250 \times 260-280$; sucker width ratio $1: 1$; pharynx 9$10 \times 8$-10; testes $280-340 \times 220-250$; ovary $140-160 \times$ 470; eggs 26-30 × 12-16.

Host: Pimelodus lateristrigus (Müller \& Troschel, 1849) (Pimelodidae).

Site: intestine.

Material deposited: CHIOC n. 33925 a-b.

Intensity of infection: one out of two fish examined was parasitized by two trematodes.

Parspina argentinensis was described by Szidat (1954) from Pimelodus clarias from Argentina. In Brazil it was referred and figured by Fortes \& Hoffmann (1985) from the intestine of Pimelodus maculatus from Guaiba estuary. Kohn \& Fróes (1986) redescribed it with new morphological data and figures from the same host and locality. We recovered specimens similar to those reported by Kohn \& Fróes (1986) from the intestine of Pimelodus lateristrigus, which represents a new host record.

\section{Neocladocystis intestinalis (Vaz,1932)}

Manter \& Pritchard, 1969 (Acanthostomidae)

Main measurements based on 8 whole-mounts: body $0.82-2.53 \mathrm{~mm}$ (1.81) long by $0.20-0.57 \mathrm{~mm}(0.47)$

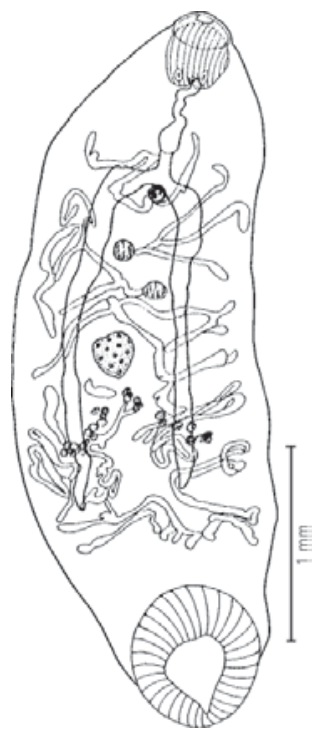

Fig. 4 - Microrchis oligovitellum. Total, ventral view. Original figure. wide; oral sucker 75-112 $(97) \times 82-123(113)$; prepharynx 15-123 (56) long; pharynx 37-75 (61) ×3056 (46); oesophagus 37-187 (100) long; ventral sucker 49-82 (69) $\times$ 49-94 (72); sucker width ratio 1:0.6-0.8 (1:0.6); testes 191-460 (354) ×75-200 (144); ovary 86266 (172) x 86-221 (138); seminal receptacle 180-187 × 67-90; eggs 28-37 (31) × 14-19 (16).

Host: Salminus maxillosus Valenciennes, 1840 (Characidae).

Site: intestine.

Material deposited: CHIOC n. 33922 a-h.

Intensity of infection: 6 out of 10 fish examined were parasitized by 1 to 120 trematodes.

Travassos et al. (1928) identified as Cladocystis trifolium (Braun, 1900) some trematodes collected from Salminus maxillosus from MogiGuaçu River. Vaz (1932) described the new species C. intestinalis from the same host from "Tietê" River and considered $C$. trifolium of Travassos et al. (1928) its synonym. Cladocystis intestinalis was transferred to Neocladocystis by Manter \& Pritchard (1969). Neocladocystis intestinalis was also referred by Travassos \& Kohn (1965) and by Kohn et al. (1985) from Salminus hilarii. Recently Pavanelli et al. (1997b) referred it also in S. maxillosus as $C$. intestinalis. We recovered specimens from the typehost, which are a little smaller than those described by Vaz (1932), and smaller and longer than specimens studied by Kohn et al. (1985). They are as large as the specimens presented by Travassos et al. (1928), with narrower eggs.

\section{Thometrema overstreeti (Brooks, Mayes \&} Thorson, 1979) Lunaschi, 1989 (Derogenidae)

Main measurements: body $3.71 \mathrm{~mm}$ long by $1.21 \mathrm{~mm}$ wide; oral sucker $480 \times 510$; ventral sucker $830 \times 820$; sucker width ratio 1:1.7; pharynx $190 \times$ 180 ; testes $390-400 \times 310-370$; ovary $200 \times 420$; eggs $42-47 \times 19-21$.

Host: Salminus maxillosus Valenciennes, 1840 (Characidae).

Site: stomach.

Material deposited: CHIOC n. 33926.

Intensity of infection: one out of ten fish examined was parasitized by one trematode.

Thometrema overstreeti was reestudied by Kohn et al. (1990) from Pimelodus maculatus and Rhamdia sp. from south of Brazil. It was also reported from different hosts in Argentina (Lunaschi, 1988; Hamann, 1986), Colombia (Brooks et al., 1979). This is the first reference of this species in Salminus maxillosus in Brazil, host already reported 
by Hamann (1986) in Argentina as Genarchella genarchella Travassos et al., 1928, and by Iannacone \& Luque (1993) in Peru.

\section{Pseudosellacotyla lutzi (Freitas, 1941) Yamaguti, 1954 (Microphallidae)}

Main measurements: body $0.66 \mathrm{~mm} \times 0.45$ mm; oral sucker $75 \times 79$; pharynx $49 \times 49$, ventral sucker $49 \times 56$; sucker width ratio 1 : 0.68 ; ovary $75 \times$ 86; testes 13-15 × 9-10; eggs 35-38 × 21-23.

Host: Hoplias malabaricus (Bloch, 1794) (Erythrinidae).

Site: intestine.

Material deposited: CHIOC n. 33929.

Intensity of infection: one out of three fish examined was parasitized by one trematode.

This species, described from $H$. malabaricus by Freitas (1941) was redescribed by Kohn et al. (1985) from the same host.

Our specimen is similar to those reported by these authors, having wider eggs (21-23 instead of 16-19).

\section{Zonocotyle bicaecata Travassos, 1948 (Zonocotylidae)}

Main measurements: body $4.73 \mathrm{~mm}$ x $1.46 \mathrm{~mm}$; oral sucker $37 \times 46$; ventral disc 2180 in diameter; oesophagus 1240 ; ovary $200 \times 230$; testis 290 in diameter; eggs 59-70 × 35

Host: Cyphocharax nagelii (Steindachner, 1881) (Curimatidae).

Site: intestine.

Material deposited: CHIOC n. 33930.

Intensity of infection: one out of five fish examined was parasitized by one trematode.

Zonocotyle bicaecata was described by Travassos (1948a) from the intestine of Steindachnerina elegans (Steindachner, 1874) (= Curimatus elegans Steindachner, 1874) from MogiGuaçu River, São Paulo State, Brazil. It was redescribed by Padilha (1978) from Cyphocharax nagelii (Steindachner, 1881) (= Curimatus nagelii) from Rio de Janeiro State, Brazil, and by Kohn et al. (1985) from Pseudocurimata plumbea (Eigenmann \& Eigenmann, 1899) from the type locality. Our specimen is similar to those studied by Kohn et al. (1985).

Besides those species we also collected specimens of:Clinostomum marginatum (Rudolphi, 1819) Braun, 1899 (immature form) from the stomach of Pseudoplatystoma corruscans (Agassiz, 1829) (Pimelodidae). Plehniella sp. from the stomach of
Hemisorubim platyrhynchos (Valenciennes, 1840) (Hypophthalmidae) and Leporinus obtusidens (Valenciennes, 1847) (Anostomidae). Rhipidocotyle sp. from the intestine of Salminus maxillosus Valenciennes, 1840 (Characidae). Saccocoelioides sp. from the intestine of Prochilodus scrofa Steindachner, 1881 (Prochilodontidae), Pterygoplichthys aculeatus (Perugia, 1891) (Loricariidae) and Schizodon knerii (Steindachner, 1875) (Anostomidae). Immature forms of Strigeidae encysted in fins of Geophagus brasiliensis (Quoy \& Gaimard, 1824) (Cichlidae).

Acknowledgments - The authors are grateful to Dr. Manuel P. de Godoy for the hosts' identification and to the directory of "Centrais Elétricas do Sul do Brasil S/A ELETROSUL", for the facilities offered during our stay in the locality of Guaíra, Paraná State.

\section{REFERENCES}

BRAY, R. A., CHAMBRIER, A. \& VAUCHER, C., 1996, Crassicutis intermedius (Szidat, 1954) n. comb. and Procaudotestis uruguayensis Szidat, 1954 (Digenea: Homalometridae) from siluriform fishes in Paraguay. Syst. Parasit., 35: 119-126.

BROOKS, D. R., MAYES, M. A. \& THORSON, T. B., 1979, Paravitellotrema overstreeti sp. n. (Digenea: Hemiuridae) from Colombian freshwater stingray Potamotrygon magdalenae Dumeril. Proc. Helminth. Soc. Wash., 46: 52-54.

ESCHMEYER, W. N., FERRARIS, C. J., HOANG, M. D. \& LONG, D. J., 1998, Catalog of Fishes. I. Species of Fishes. pp. 25-1820. California Academy of Sciences, San Francisco, 2905p.

FORTES, E. \& HOFFMANN, R. P., 1985, Contribuição ao conhecimento dos parasitos de peixes do estuário do Guaíba, no Estado do Rio Grande do Sul. IV. Trematódeos digenéticos. Rev. Bras. Med. Vet., 7(7): 209-210.

FREITAS, J. F. T., 1941, Sellacotyla lutzi n. sp. trematódeo parasito de Hoplias malabaricus Bloch. Ann. Acad. Brasil. Sci., 13: 17-19.

HAMANN, M. I., 1986, Halipegus ovocaudatus (Vulpian, 1859) Looss, 1899 (Hemiuridae, Halipeginae) parasita de Rana esculenta Linne, de Europa y Genarchella genarchella Travassos, Artigas \& Pereira, 1928 (Hemiuridae, Halipeginae) parasita de Salminus maxillosus Valenciennes, 1840 del sudeste de América del Sur. Anatomia y posición sistemática. Physis, Buenos Aires secc. B, 44(106): 19-24.

HAMANN, M. I., 1989, Genarchella Travassos, Artigas y Pereira, 1928 (Digenea, Hemiuridae) parasitos de peces de agua dulce del río Paraná, provincia de Corrientes, República Argentina. I: Anatomia y posición sistemática. II.: Contribuciones ecológicas. Physis, Buenos Aires secc. B, 47(112): 15-30. 
IANNACONE, J. A. \& LUQUE, J. L., 1993, New records of helminths parasitic on Peruvian Amazonian fishes (Osteichthyes). Rev. Biol. Trop., 41(2): 303-305.

KOHN, A. \& FERNANDES, B. M. M., 1988, Revision of the Brazilian species of the genus Halipegus Looss, 1899 (Trematoda: Derogenidae). Syst. Parasit., 11: 129-137.

KOHN, A. \& FERNANDES, B. M. M., 1994 Rhipidocotyle gibsoni $\mathrm{n}$. sp. from a Brazilian freshwater fish and Rhipidocotyle froesi $\mathrm{n}$. sp. for $R$. baculum (Linton, 1905) of Eckmann (1932) (Bucephalidae; Digenea). Mem. Inst. Oswaldo Cruz, 89(4): 567-570.

KOHN, A., FERNANDES, B. M. M. \& BAPTISTA-FARIAS, M. F. D., 1997, Redescription of Prosthenhystera obesa (Diesing, 1850) (Callodistomidae, Digenea) with new host records and data on morphological variability. Mem. Inst. Oswaldo Cruz, 92(2): 171-179.

KOHN, A., FERNANDES, B. M. M. \& GIBSON, D. I., 1999, Chalcinotrema thatcheri n. sp. (Digenea: Haploporidae) from Brazilian freshwater fishes, a redescription of $C$. ruedasueltensis Thatcher, 1978 and comments on the validity of the genus. Syst. Parasit., 44: 211-215.

KOHN, A., FERNANDES, B. M. M., GIBSON, D. I. \& FRÓES, O. M., 1990, On the Brazilian species of halipegine genera (Trematoda: Derogenidae) from fishes, with new morphological data, hosts and synonyms. Syst. Parasit., 16: 201-211.

KOHN, A., FERNANDES, B. M. M., MACEDO, B. \& ABRAMSON, B., 1985, Helminths parasites of freshwater fishes from Pirassununga, SP, Brazil. Mem. Inst. Oswaldo Cruz, 80(3): 327-336

KOHN, A. \& FRÓES, O. M., 1986, Saccocoelioides godoyi n. sp. (Haploporidae) and other trematodes parasites of fishes from the Guaiba estuary, RS, Brazil. Mem. Inst. Oswaldo Cruz, 81(1): 67-72.

LUNASCHI, L. I., 1987, Helmintos parasitos de peces de agua dulce de la Argentina VI. Sobre una nueva especie del género Microrchis Daday, 1907 (Trematoda, Paramphistomidae). Neotropica, 33(89): 37-40.

LUNASCHI, L. I., 1989, Helmintos parasitos de agua dulce de la Argentina. X. Tres nuevas especies del género Thometrema Amato, 1968 (TrematodaDerogenidae). Neotropica, 34(91): 23-32.

MANTER, H. W., 1936, Some trematodes of Cenote fish from Yucatan. Carn. Inst. Wash. Publ., 457: 431-439.

MANTER, H. W. \& PRITCHARD, M. H., 1969, Some digenetic trematodes of Central Africa chiefly from fishes. Revue. Zool. Bot. Afr., 80(1/2): 51-61.

MORAVEC, F., KOHN, A. \& FERNANDES, B. M. M. 1992a, Three new species of oxyuroid nematodes, including two new genera, from freshwater catfishes in Brazil. Syst. Parasit., 21: 189-201.

MORAVEC, F., KOHN, A. \& FERNANDES, B. M. M., 1992b, Neoparaseuratum travassosi n. g., n. sp. (Nematoda: Quimperiidae), a new parasite from thorny catfish Pterodoras granulosus in Brazil. Mem. Inst. Oswaldo Cruz, 87, Suppl. I: 145-150.
MORAVEC, F., KOHN, A. \& FERNANDES, B. M. M., 1992c, Nematode parasites of fishes of the Paraná River, Brazil. Part 1. Trichuroidea, Oxyuroidea and Cosmocercoidea. Folia Parasitol., 39: 327-353.

MORAVEC, F., KOHN, A. \& FERNANDES, B. M. M., 1993a, Nematode parasites of fishes of the Paraná River, Brazil. Part 2. Seuratoidea, Ascaridoidea, Habronematoidea and Acuarioidea. Folia Parasitol., 40: 115-134.

MORAVEC, F., KOHN, A. \& FERNANDES, B. M. M. 1993b, Nematode parasites of fishes of the Paraná River, Brazil. Part 3. Camallanoidea and Dracunculoidea. Folia Parasitol., 40: 211-229.

PADILHA, T. N., 1978, Caracterização da família Zonocotylidae com redescrição de Zonocotyle bicaecata Travassos, 1948 e descrição de um novo gênero (Trematoda: Digenea). Rev. Brasil. Biol., 38(2): 415-429.

PAVANELLI, G. C., EIRAS, J. C. \& GUIDELLI, G. M., 1997a, Histopatology of the infection by Microrchis oligovitellum Lunaschi, 1987 (Trematoda - Paramphistomidae) in Parauchenipterus galeatus (Linnaeus, 1766). Rev. Unimar, 19(2): 473-478.

PAVANELLI, G. C., MACHADO, M. H. \& TAKEMOTO, R. M. B., 1997b, Fauna helmíntica de peixes do Rio Paraná, região de Porto Rico, Paraná. pp. 307-329. In: A. E. A. M. Vazzoler, A. A. Agostinho \& N. S. HAHN, 1997, A planície de inundação do alto Rio Paraná: aspectos físicos, biológicos e socioeconômicos. Ed. Univ. Est. de Maringá, 460p.

SCHOLZ, T., PECH-EK, M. C. F. \& RODRIGUEZ-CANUL, R., 1995a, Biology of Crassicutis cichlasomae, a parasite of cichlid fishes in Mexico and Central America. J. Helminth., 69: 69-65.

SCHOLZ, T., VARGAS-VASQUEZ, J. \& SALGADOMALDONADO, G., 1995b, Revision of Genarchella species (Digenea: Derogenidae) parasitizing freshwater fishes in Mexico and Central America. J. Nat. Hist., 29: 1403-1417.

SZIDAT, L., 1954, Tremátodes nuevos de peces de agua dulce de la República Argentina y intento para aclarar su carácter marino. Rev. Inst. Nac. Invest. Ci. Nat. Mus. Argen. Ci. Nat. Bernardino Rivadavia, C., Zool., 3(1): 85p.

TRAVASSOS, L., 1948a, Contribuição ao conhecimento dos helmintos dos peixes d'água doce do Brasil. I. Zonocotyle bicaecata n. g. n. sp. (Trematoda, Aspidogastridae). Mem. Inst. Oswaldo Cruz, 45(2): 513-516.

TRAVASSOS, L., 1948b, Contribuição ao conhecimento dos helmintos dos peixes d'água doce do Brasil. II. Iheringtrema iheringi n. g., n. sp. (Trematoda, Heterophyidae). Mem. Inst. Oswaldo Cruz, 45(2): 517-520.

TRAVASSOS, L., ARTIGAS, P. \& PEREIRA, C., 1928, Fauna helmintológica dos peixes de água doce do Brasil. Arch. Inst. Biol., 1: 5-68.

TRAVASSOS, L. \& KOHN, A., 1965, Lista dos helmintos parasitos de peixes encontrados na Estação Experimental de Biologia e Piscicultura de Emas, Pirassununga, Estado de São Paulo. Pap. Avuls. Dep. Zool., São Paulo, 17(5): 35-52.

VAZ, Z., 1932, Contribuição ao conhecimento dos trematóides de peixes fluviais do Brasil. São Paulo, 47p. 